\section{EMBRYRIDDLE Aeronautical University}

SCHOLARLY COMMONS
International Journal of Aviation, Aeronautics, and Aerospace

\title{
Passengers from India and the United States Have Differential Opinions about Autonomous Auto-Pilots for Commercial Flights
}

\author{
Stephen Rice \\ Florida Institute of Technology, srice@fit.edu \\ Keegan Kraemer \\ keegankraemer@gmail.com \\ Scott R. Winter \\ Florida Institute of Technology, scott.winter@mac.com \\ Rian Mehta \\ Florida Institute of Technology, rianmehta91@gmail.com \\ Victoria Dunbar \\ Florida Institute of Technology, dunbarv@fit.edu \\ Timothy G. Rosser \\ Florida Institute of Technology, trosser@fit.edu \\ Julie C. Moore \\ Florida Institute of Technology, moore@fit.edu
}

Follow this and additional works at: https://commons.erau.edu/ijaaa

Part of the Other Psychology Commons, and the Other Social and Behavioral Sciences Commons

\section{Scholarly Commons Citation}

Rice, S., Kraemer, K., Winter, S. R., Mehta, R., Dunbar, V., Rosser, T. G., \& Moore, J. C. (2014). Passengers from India and the United States Have Differential Opinions about Autonomous Auto-Pilots for Commercial Flights. International Journal of Aviation, Aeronautics, and Aerospace, 1(1). https://doi.org/ 10.15394/ijaaa.2014.1004

This Article is brought to you for free and open access by the Journals at Scholarly Commons. It has been accepted for inclusion in International Journal of Aviation, Aeronautics, and Aerospace by an authorized administrator of Scholarly Commons. For more information, please contact commons@erau.edu. 
Many studies and articles indicate the differences in culture between Indian and American culture. However, very few studies investigate the differences in comfort, trust, and willingness to accept automated technologies, specifically, when flying in a commercial aircraft. The current study focuses on perceptions of participants from India and the United States on comfort, trust, and willingness toward remote controlled and autonomous auto-pilots (defined as fully autonomous computers that operate without interference from human pilots) to be used for commercial flight operations.

\section{Comparisons of Culture: India and The United States}

On average, 50 million passengers fly in and out of India on an annual basis (Carrerio, n.d.). India is rapidly emerging in the aviation market and positioned to transform the flight and automated industries (Couchen, \& Lieching, 2008). As a possible challenge to this growth, as of 2010, the United States has seen a decrease in year-to-date commercial pilot certificate issuance by 29\% (Aircraft Owners and Pilot's Association, 2010). This reduction in pilot certification may be an indicator of a fore looming pilot shortage. Boeing (2013) reported the need for 498,000 new commercial airline pilots worldwide in the next 20 years. Clearly there is a disconnect as passenger numbers grow and the number of pilots to fly them is decreasing.

Paralleling the potential pilot shortage is an increase in technological advances in flight technology. Along with a cultural influence, public perception of automated flight capabilities may directly influence aircraft automation and design. Airlines all over the world are seeing an increase in advanced technologies by passengers using both Apple and Android applications through wifi technologies (airlinetrends.com, 2013). Airline customers are able to communicate in real time with the flight crew, be fully engaged with in-flight entertainment, and generate ancillary revenue for the airline buy purchasing products in-flight. Although this may not directly relate to automated flight capabilities and customers' feelings towards them, it does illustrate that society is becoming ever more accepting of advancing technologies. One's culture, however, can play an integral role in this acceptance and interaction with new technologies.

Culture is defined by Helmreich (2000) as "the shared norms, values, and practices associated with a nation, organization, or profession" (p. 134). Since culture can exist on many levels it is important to identify that the following paper will look at the national level of culture difference between India and the United States. Two key parameters that will be analysed for differences between these two cultures are individualism versus collectivism and uncertainty avoidance. 
The differences between American and Indian culture in regards to the individualistic and collectivist views are widely documented. Those in collectivist cultures, such as India, hold an interdependent view of the self (Markus \& Kitayama, 1991). Furthermore, cultural background influences an individual's propensity to trust (Hofstede, 1980). It has also been shown that extraverts are more willing to trust other individuals, than are introverts (Gaines et al., 1997; Omodei \& McLenna, 2000; Shikishima, Hiraishi, \& Ando, 2006). Even from an early age, individuals in collectivist cultures are taught to totally trust without question (Wu \& Jang, 2008). Those with allocentric tendencies exhibit a higher concern for others' opinions in their decision-making. For example, an individual of Indian descent may show a higher concern for their colleagues' well-being over their own, for fear a contradictory decision would offend them. Furthermore, allocentric individuals tend to base their decisions on collectivist values, with a community-based mindset, taking others' interests into higher regard over their own.

According to Hofstead's Cultural Values by Nation Index, India scored a 48 out of 100 on the individualistic versus collectivist dimension (Robbins and Judge, 2009). This indicates that India is mostly collectivist, but may also exhibit individualist features, whereas Guatemala, for example, scored the lowest at a six making it a highly collectivist culture. The United States, on the other hand, scored the highest at a 91, indicating that the US is an extremely individualistic culture.

One other dimension that may assist in determining the meaning behind a particular culture's feelings regarding automated flight technologies is uncertainty avoidance. Robbins and Judge (2009) define uncertainty avoidance as the "extent to which a society feels threatened by uncertain and ambiguous situations and tries to avoid them" (p. 125). Advancing technologies that utilize automated or remote-controlled aircrafts may be deemed as ambiguous or uncertain situations. Looking again at Hofstede's Cultural Values by Nation Index, India scored a 40 on this dimension, while the US scored a 46 out of 100. Granted the difference between the two is not as drastic, there is still a distinct change in uncertainty avoidance between the two countries. Those of Indian descent are more likely to take risks with ambiguous outcomes than those individuals from the US.

\section{Expansion of Automated Technologies and Trust}

Over the past few decades, there has been an exponential growth in automated technologies (Rice, 2009). Reliability of this automation can improve task performance (Wickens \& Dixon, 2007). Furthermore, automation of certain systems can allow for successful multitasking. Parasuraman, Sheridan, and Wickens (2000) outline the four different stages 
of automation, which is very similar to human information processing; information synthesis, diagnosis, selection, and execution. An individual's ability to trust is a psychological state, which in this case is influenced by a variety of varying factors. If an individual has the ability to access raw data, they then make the choice as to whether or not to blindly accept or reject the automation's recommendation (Sorkin \& Woods, 1985). When this automation fails, both operator trust and dependence are affected.

Levels of human comfort, trust, and willingness to utilize automated technologies should be taken into serious consideration as its use can have life-and-death implications (Merritt \& Ilgen, 2008). Individuals will use machines that they trust more often. An individual's dispositional trust can be influenced by their tendency to trust automation through "automation induced compliancy potential" (p. 196). These automated technologies carry machine characteristics that are linked to perceptions of trust: competence, predictability, and dependability. A user's propensity to trust these automated machines and their characteristics interact to affect subsequent ratings of trust.

As mentioned previously, there have been very few studies that investigated levels of trust in auto-pilots. One such study by Hughes, Rice, Trafimow, and Clayton (2009) examined passengers' trust in human versus auto-pilots, and reported that even when given price discounts, passengers held more negative attitudes towards the auto-pilot compared to the human pilot.

\section{Current Study}

The current study expands on previous research in three ways. First, while Hughes et al. (2009) compared human pilots to auto-pilots, they did not examine attitudes towards remote-controlled (RC) flights (i.e. when a human pilot flies the aircraft from a remote location). These types of flights are commonplace now in the military and civilian surveillance (e.g. drones). It may only be a matter of time before they are also used for other types of aviation, perhaps in response to the forecasted demand for pilots. Second, previous studies did not examine cultural differences in attitudes. The current study addresses this gap by comparing Indian to American consumers. Third, the previous studies did not compare attitudes as a function of who is riding in the aircraft. The current study addresses this gap by telling participants that either they themselves, their child, or their work colleague would be on the flight. The research question under investigation was: How will participant's level of comfort, trust, and willingness to fly on an aircraft controlled by human pilots, fully autonomous, or remotely controlled vary based on culture (Indian vs. US) and who is on-board the aircraft (themselves, their child, a colleague)?

We hypothesized the following: 
$\mathrm{H}_{1}$ : In general, participants would show more negative attitudes towards the auto-pilot (defined as fully autonomous computers that operate without interference from human pilots) and RC pilot compared to the human pilot in the cockpit.

$\mathrm{H}_{2}$ : Indian participants would be more forgiving in their attitudes towards auto-pilots and RC pilots compared to American participants given the collectivist tendency to trust more in something that authorities have deemed safe.

$\mathrm{H}_{3}$ : In general, participants would be more negative in their attitudes when their child was involved, but less negative when their work colleague was involved, a predicated effect based on self-preservation.

\section{Method}

\section{Participants}

One hundred and four participants (53 females) from the United States took part in the study. The mean age was $31.01(S D=9.75)$. Ninety-seven participants (33 females) from India took part in the study. The mean age was $31.34(S D=9.11)$.

\section{Materials and Recruitment}

The study was presented online using FluidSurveys @ ${ }^{\circledR}$. Participants were recruited via Amazon's @ Mechanical Turk @ (MTurk). MTurk is a global online service that enables participants (Turkers) to participate in Human Intelligence Tasks (HITs) in exchange for monetary compensation. Participation in any HIT is voluntary and anonymous.

\section{Procedure}

Participants first signed an electronic consent form. They were then asked to imagine that there was a flight between two major cities that needed to be taken in order to attend a function in the other city. Participants were told that either they themselves, their child, or their work colleague would be on the flight. They were also told that the flight would be piloted with either a human pilot, an auto-pilot (fully autonomous machines that operate without interference with human pilots), or a human pilot in a ground station using a remote control system (e.g. drone). After each scenario, participants were asked how comfortable they would feel in the scenario, how much they would trust the entity piloting the aircraft, and how willing they would be to participate in the scenario. These responses were given on a 7-point Likert 
scale from Extremely Uncomfortable/Distrust/Unwilling (-3) to Extremely Comfortable/Trust/Willing $(+3)$. A neutral response of zero was permitted. Upon completion of the study, participants were debriefed and paid.

\section{Design}

There were two independent variables with 3 levels each. Thus, this was a $3 \times 3$ within-participant factorial design.

\section{Results}

First, the three dependent variables (trust, comfort, and willingness) were subjected to a Cronbach's Alpha test to determine the level of internal consistency. The values ranged from .81 to .89 . Due to the high internal consistency between the answers, the data were averaged into one score per participant.

A $3 \times 3 \times 2$ ANOVA was conducted on the data using Country as a between-participant factor and TypeOfPilot and Passenger as withinparticipant factors. There were main effects of Country, $F(1,199)=22.89, p<$ $.001, \eta p^{2}=.10$, of TypeOfPilot, $F(2,398)=469.84, p<.001, \eta p^{2}=.70$, and Passenger, $F(2,398)=65.49, p<.001, \eta p^{2}=.25$. There was a 3-way interaction, $F(4,796)=5.75, p<.001, \eta p^{2}=.03$, along with 2-way interactions between Country and TypeOfPilot, $F(2,398)=26.81, p<.001$, $\eta p^{2}=.12$, Country and Passenger, $F(2,398)=16.04, p<.001, \eta p^{2}=.08$, and TypeOfPilot and Passenger, $F(4,796)=14.31, p<.001, \eta p^{2}=.07$.

Overall, participants were more comfortable/trusting/willing with the human pilot compared to the other two conditions. Figure 1 reveals that, in

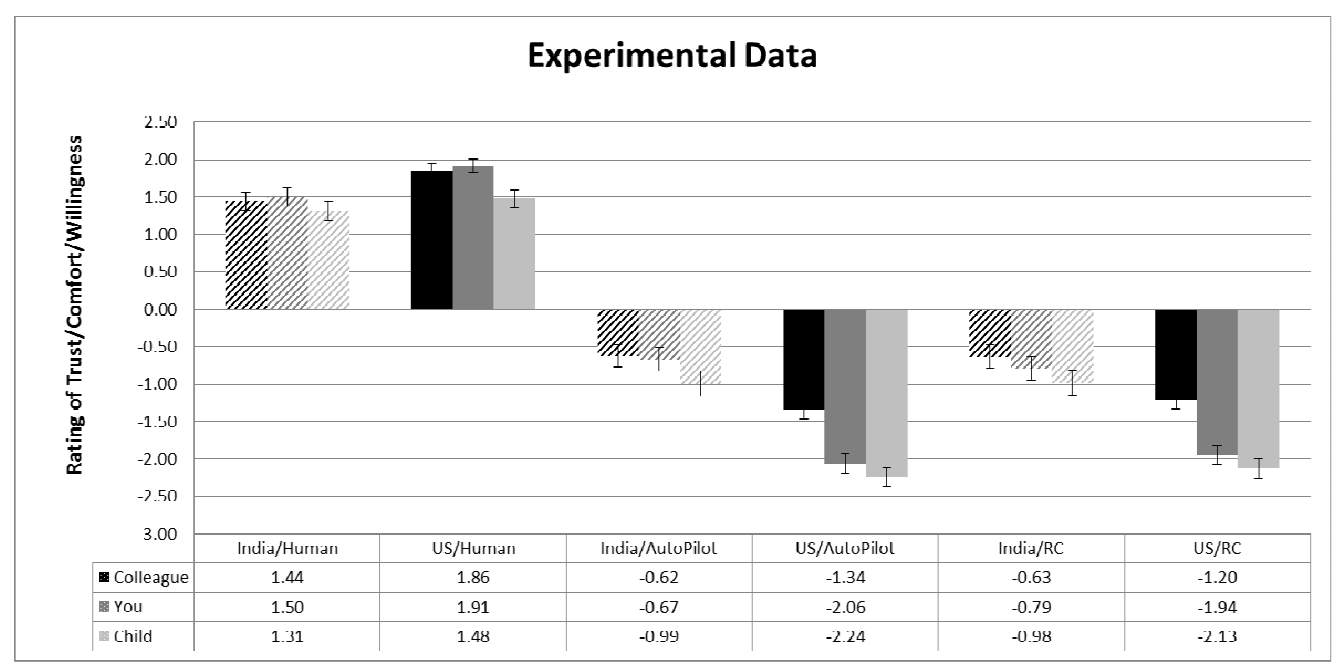

Figure 1. Data from the study. SE bars are included in the figure. The thatched bars represent Indian data. 
general, the US participants tended to be more positive about the human pilot ( $p s<.05)$ except in the Child condition, and much more negative about the autopilot and RC pilot ( $p s<.01$ ), compared to Indian participants. Participants appeared to be more negative about having their child in the situation, although these post hoc comparisons were not all statistically significant.

\section{Discussion}

The purpose of this study was to investigate the cultural differences between Americans and Indians, with respect to their comfort, trust, and willingness toward remote controlled and fully autonomous commercial flight operations. The study was designed to gauge a person's perception with respect to whether the aircraft was being piloted with a human pilot, a completely automated aircraft (no human involved), or a human pilot in a ground station using a remote control system. It adds a secondary cultural and sociological layer by asking the same question with respect to who was flying on board the aircraft: themselves, their child, or their colleague.

The first hypothesis stated that in general, participants should show more negative attitudes towards the completely automated aircraft and RC operated aircraft compared to the human pilot in the cockpit. The results from both American and Indian demographics support this hypothesis in all three categories of comfort, trust, and willingness. Both demographics scored the human pilot in the cockpit with a high positive rating, and scored the completely automated aircraft and the RC operated aircraft with a negative rating. What is interesting to note is that even though the two automated scenarios were expected to score lower than the human pilot, the fact that, almost unanimously across both demographics, there was no scoring difference between the completely automated aircraft and the RC operated aircraft. The study indicates that people are as unwilling, untrustworthy, and uncomfortable with RC operated aircraft as with the completely automated aircraft, even though the RC operated aircraft involves human in the loop control and is not solely operated by computers. The findings suggest this has no effect on people's opinions of the situation and could be of valuable use to airlines that are looking to make changes in terms of making the pilot an obsolete tool in the cockpit. If people are not willing to trust the automation, it could have severe negative effects on airline revenue across the globe.

Our second hypothesis states that Indian participants would be more forgiving in their views towards completely automated aircrafts and RC operated aircraft compared to American participants given Indian culture's collectivist tendency to trust more in something that authorities have deemed safe (Wu \& Jang, 2008). As predicted, the Indian participants were less extreme in their views of each of the three dependent variables than their American counterparts. The Americans trusted the human pilot more than the 
Indian participants, but consecutively trusted the auto-pilot aircraft and RC operated aircraft less than the Indians. The hypothesis is supported by the fact that the cause of these differences between the two cultures is quite possibly rooted in the differences between collectivist and individualistic societies. The collectivist Indian culture is more willing and trustworthy of the automation than the American culture, due to the belief and trust in one's government to only sanction such a technology if it is deemed safe for the public (Wu \& Jang, 2008). The collectivist nature of the Indian culture teaches to trust other individuals in the community more willingly.

The Indian culture, being collectivist, is also ingrained with certain subconscious traits of teaching people not to be extremists or ones to challenge the status quo (Wu \& Jang, 2008). They are petitioned not to rebel or be ones to push boundaries, and rather be a culture more heavily based on conformity, mainly due to a rich emphasis on traditions and religion. It is possibly for this reason that on most scales, Indian participants will rank closer to the mean/median on both sides of the topic, rather than be the extremists that seek to be ones to question the norm.

Another possible explanation may be uncertainty avoidance - the extent to which a society feels threatened by uncertain and ambiguous situations and tries to avoid them. Here again the findings show Americans are less likely to accept uncertainty than the sample of participants from India. It is not as drastic as the difference between individualistic and collectivist, but it is a distinct difference. The US scored a 46 out of 100 while India scored a 40 out of 100 on an uncertainty avoidance measure (Robbins \& Judge, 2009). This shows that Americans are more risk averse. Indians are more likely to take risks with ambiguous outcomes. The data from this study indicates that those from an Indian culture would be more willing to be a passenger on an aircraft that does not have a pilot at the helm than those from an American culture.

This finding could be of great value to the future of automation in the aviation industry. There has been a heavy surge in the field of automation in aviation, but the restraints lie in the trust and willingness of the general public to accept such drastic changes. These findings suggest that if airlines would attempt to move toward more automated aircraft with less human involvement, varying cultural traits may have to be addressed, and it appears unlikely, at least from this study, that acceptance of this technology would be uniform across various cultures. If these technologies were to be approved by ICAO and the DGCA (Directorate General of Civil Aviation, Government of India), the Indian culture may become viable testing grounds for future aviation advancements. 
The last hypothesis stated that in general, participants would be more negative in their attitudes when their child was involved, but less negative when their work colleague was involved, a predicated effect based on selfpreservation. This is clearly supported by the findings amongst both demographics. In all three pilot type scenarios, across all three dependent variables (comfort, trust, and willingness), the participants were less comfortable, trusting, and willing to be on the flight compared to their colleague being on board. What was also predicted, and supported by the findings, was that the participants would be even more hesitant if it were their child on board, than themselves or their colleague. These findings are most likely linked to the innate characteristic of self-preservation, as well as, the fear and primal instinct to protect one's own offspring.

\section{Practical Implementations}

As stated earlier, there are certain real-world applications of the findings from this study. It helps make the airlines aware of the public's idea and trust towards unmanned or remote controlled automated flight, allowing them to evaluate whether this is a viable option for the future and what challenges may exist before implementation of such technologies. Airlines may experience some drop in revenue if they decide to implement these new technologies, due to the reservations of the public. The knowledge of certain markets being more willing to board flights with more automation may become useful in identifying potential testing grounds for the trust in new automation.

The findings of this study may help guide the future actions of the airline industry in terms of gauging public opinion. If pursued, these findings could help airlines become more cost efficient, which in turn will lead to potential cheaper airfares enjoyed by the general public. If the future studies in this field are able to detect where the general public is willing to make compromises in personal reservations, it could mean explosive growth for the profits of the airlines, and the industry as a whole. More profitable airlines will also indirectly lead to better service offered to the passengers.

\section{Limitations and Recommendations for Future Research}

The future of automation seems almost limitless, and there is vast array of information yet to be comprehended about this field. This study only measures three dependent variables (comfort, trust, and willingness) based off of three different aircraft types. The study can be taken further to include several variations of the pilot control types. The future aim should be to find the drop off point in the public's trust after which the level of automation is unacceptable. If we are able to find the tipping point, the economics of the aviation industry could be heavily altered. One potential avenue to explore 
could be where the participants are presented with an option of a sole pilot in the cockpit versus the conventional two pilot set up. Consecutively research could pursue an option where there is a fully trained human auto-pilot monitor placed in the cockpit, whose sole purpose would be to intervene in case of emergencies.

Certain limitations also exist by way of the small pool of dependent variables. This could be increased to a larger base, to gauge more clearly the full effect of this theory. This study also chooses to neglect any external factors that could cause such decisions to be made, such as socio-economic influences. Although Hughes et al. (2009) looked at monetary differences, more research should be conducted to examine if people's answers may be swayed by the influence of monetary conditions, and research could be conducted to see if people are more likely to become accepting of automation if it resulted in large financial savings in air travel. The breaking point in financial savings versus personal reservations would be the next step in the study of this theory. The study was also limited to participants that complete online human intelligence tasks (HITs), and the possible technological biases associated with this type of population. The small sample size also limits the generalizability of the findings. A final limitation to the study was that some participants might not have had a child. This lack of having a child may have influenced participant's answer to questions related to their children being the passenger on the hypothetical flights.

\section{Conclusion}

This study has successfully displayed the cultural differences and attitudes towards completely autonomous automation in commercial aviation. Valuable data has been collected to gauge whether the general public is willing to accept further advancements in automation as part of their regular travel plans. It has also been shown that the collectivistic nature of certain cultures has a distinct impact upon their comfort, trust, and willingness towards automated commercial aviation, and therefore is more likely to accept the same as compared to their individualistic counterparts in other cultures. The data from this study supports the initial hypotheses, and therefore has demonstrated the need for future studies in this field to explain these phenomena in more detail. These findings are of significant practical use for determining the future growth and direction adopted by the commercial aviation industry. Only once we are able to find the balance between human interaction and automation, will it become a viable way of the future, and until then, the general public's view of autonomous automation will be a leading deterrent in this field. 


\begin{abstract}
Author Bios
Dr. Stephen Rice is an Associate Professor at Florida Institute of Technology. He received his Ph.D. in Human Factors from the University of Illinois at UrbanaChampaign in 2006. His research interests include aviation psychology, automation, trust, stigmas, and human performance.
\end{abstract}

Keegan Kraemer is a doctoral candidate at Walden University with an expected graduation date of 2015. She completed both her B.S. in psychology at Walden, as well as her M.S. in Organizational Psychology from Walden University. Her research interests include cultural factors of stigmas and stereotypes.

Dr. Scott R. Winter is an Assistant Professor at the Florida Institute of Technology. He completed his Ph.D. in Aviation Technology from Purdue University in 2013. His research interests have centered on decision-making, pilot training, and aviation human factors.

Mr. Rian Mehta is a graduate student at the Florida Institute of Technology pursuing a Master's degree in Aviation Safety. He received his Bachelor's Degree in Aviation Management with Flight from the Florida Institute of Technology in 2013. His research interests include automation, pilot fatigue, and decision-making in the cockpit.

Victoria Dunbar is an Assistant Professor at Florida Institute of Technology. She completed her master's degree in Human Resource Development at Indiana State University in 2008. She is currently pursuing her PhD in Aviation Science at Florida Institute of Technology. Her research interests include pilot training, pilot complacency, and aviation human factors.

Mr. Timothy G. Rosser is an assistant Professor at Florida Institute of Technology. He received his MBA in Finance from Marywood University in 2004. His research interests include business best practices, pilot training and pilot operations.

Julie Moore is an Assistant Professor at the Florida Institute of Technology, and an F-16 Instructor Pilot in the US Air Force Reserves. She completed her MS in Environmental Studies from Green Mountain College in 2012. Her research interests include aviation human factors, aviation safety, and remote sensing applications for unmanned aerial systems. 


\section{References}

Aircraft Owners and Pilots Association (AOPA). (2010). AOPA general aviation trends report fourth quarter 2010. Retrieved from http://www.aopa.org/About-AOPA/Statistical-ReferenceGuide/General-Aviation-Trends.aspx

Airlinetrends.com. (2013). [Online newsletter, August 25, 2013]. Retrieved from http://www.airlinetrends.com/

Boeing. (2013). Current market outlook 2013-2032. Retrieved from http://www.boeing.com/boeing/commercial/cmo/pilot_technician_outl ook.page

Carrerio, H. (n.d.). About airlines in india. Retrieved from http://traveltips.usatoday.com/airlines-india-17997.html

Couchen, W., \& Lieching, J. (2008). The moderating role of referent of focus on purchase intent for consumers with varying levels of allocentric tendency in a collectivist culture. Journal Of International Consumer Marketing, 20(3/4), 5-22. doi:10.1080/08961530802129128.

Gaines. S. O.. Panter. A.T, Lyde. M. D,. Steers. W N,, Rusbult. C, E, Cox, C. L., et al. 1997). Evaluating the complexity of interpersonal traits and the manifestation of interpersonal traits in interpersonal trust. Journal of Personality \& Social Psychology. 73, 610-623.

Helmreich, R. L. (2000). Culture and error in space: Implications from analog environments. Aviation, Space, and Environmental Medicine, 71(911), 133-139.

Hofstede, G. (1980). Motivation, leadership and organization: Do american theories apply abroad? Organizational Dynamics, 9(1), 42-63.

Hughes, J., Rice, S., Trafimow, D. \& Clayton, K. (2009). The automated cockpit: A comparison of attitudes towards human and automated pilots. Transportation Research Part F: Traffic Psychology and Behaviour, 12(5), 428-439.

Markus, Hazel Rose and Shinobu Kitayama. 1991, Culture and the self: Implications for cognition, emotion, and motivation. Psychological Review, 98(2), 224-253. 
Merritt, S. M., \& Ilgen, D. R. (2008). Not all trust is created equal: Dispositional and history-based trust in human-automation interactions. Human Factors, 50(2), 194-210. doi:10.1518/001872008X288574.

Omodei, M. M, \& McLennan. J. (2000). Conceptualizing and measuring global interpersonal mistrust-trust, 7, 140. 279-294.

Parasuraman, R., Sheridan, T. B., \& Wickens, C. D. (2000). A model for types and levels of human interaction with automation. IEEE Transactions on Systems, Man, and Cybernetics, 30, 286-297.

Rice, S. (2009). Examining single- and multiple-process theories of trust in automation. Journal Of General Psychology, 136(3), 303-319.

Robbins, S. P. \& Judge, T. A. (2009). Organizational behavior (13 ${ }^{\text {th }}$ Ed.). Upper Saddle River NJ: Prentice Hall.

Shikishima. C Hiraishi. K,. \& Ando. J. (2(X)6). Genetic and environmental influences on general trust: A test of a theory of tra.st with behavioral genetic and evolutionary psychology approaches. Japanese Journal of Social Psychology. 22, 48-57.

Sorkin, R. D., \& Woods, D. D. (1985). Systems with human monitors, a signal detection analysis. Human-Computer Interaction, 1, 49-75.

Wickens, C. D., \& Dixon, S. R. (2007). The benefits of imperfect diagnostic automation: A synthesis of the literature. Theoretical Issues in Ergonomic Science, 8, 201-212. 